\title{
Discrete and broadband electron acceleration in Jupiter's powerful aurora
}

\author{
B. H. Mauk ${ }^{1}$, D. K. Haggerty ${ }^{1}$, C. Paranicas ${ }^{1}$, G. Clark $^{1}$, P. Kollmann ${ }^{1}$, A. M. Rymer ${ }^{1}$, S. J. Bolton², S. M. Levin ${ }^{3}$, A. Adriani ${ }^{4}$, \\ F. Allegrini ${ }^{2,5}$, F. Bagenal ${ }^{6}$, B. Bonfond ${ }^{7}$, J. E. P. Connerney ${ }^{8}$, G. R. Gladstone ${ }^{2}$, W. S. Kurth ${ }^{9}$, D. J. McComas ${ }^{2,10} \&$ P. Valek ${ }^{2}$
}

The most intense auroral emissions from Earth's polar regions, called discrete for their sharply defined spatial configurations, are generated by a process involving coherent acceleration of electrons by slowly evolving, powerful electric fields directed along the magnetic field lines that connect Earth's space environment to its polar regions ${ }^{1,2}$. In contrast, Earth's less intense auroras are generally caused by wave scattering of magnetically trapped populations of hot electrons (in the case of diffuse aurora) or by the turbulent or stochastic downward acceleration of electrons along magnetic field lines by waves during transitory periods (in the case of broadband or Alfvénic aurora $)^{3,4}$. Jupiter's relatively steady main aurora has a power density that is so much larger than Earth's that it has been taken for granted that it must be generated primarily by the discrete auroral process $^{5-7}$. However, preliminary in situ measurements of
Jupiter's auroral regions yielded no evidence of such a process ${ }^{8-10}$. Here we report observations of distinct, high-energy, downward, discrete electron acceleration in Jupiter's auroral polar regions. We also infer upward magnetic-field-aligned electric potentials of up to 400 kiloelectronvolts, an order of magnitude larger than the largest potentials observed at Earth ${ }^{11}$. Despite the magnitude of these upward electric potentials and the expectations from observations at Earth, the downward energy flux from discrete acceleration is less at Jupiter than that caused by broadband or stochastic processes, with broadband and stochastic characteristics that are substantially different from those at Earth.

There were several surprises that came out of the first perijove (PJ1) encounter (on 27 August 2016) of NASA's Juno spacecraft with the low-altitude regions of Jupiter's polar auroral regions. Magnetic a

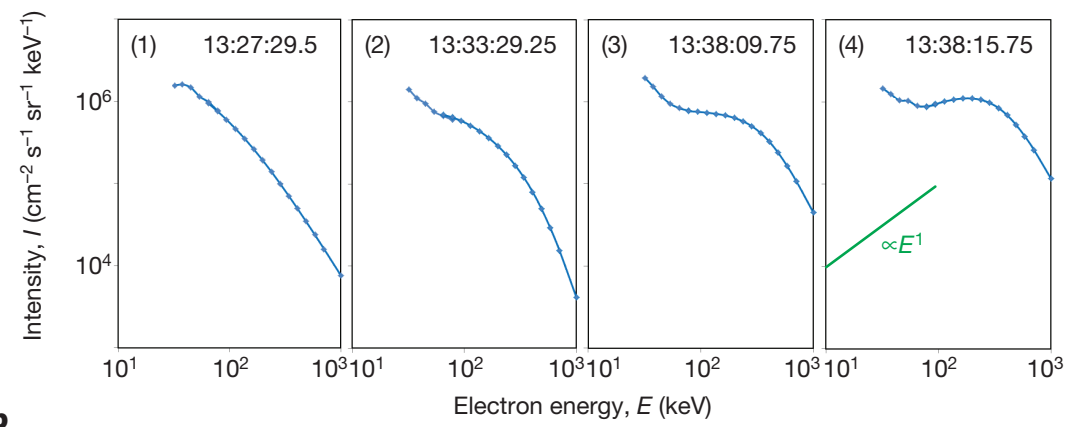

b

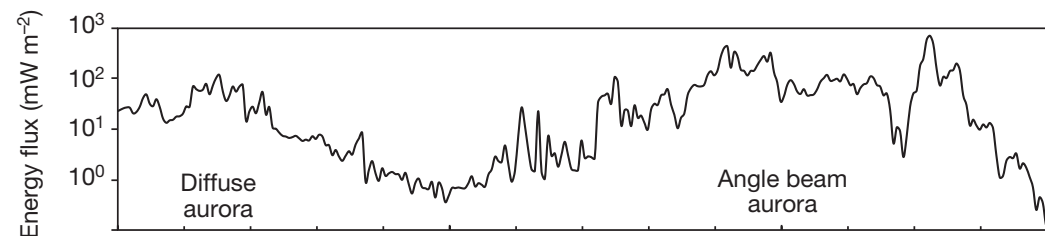

(1)

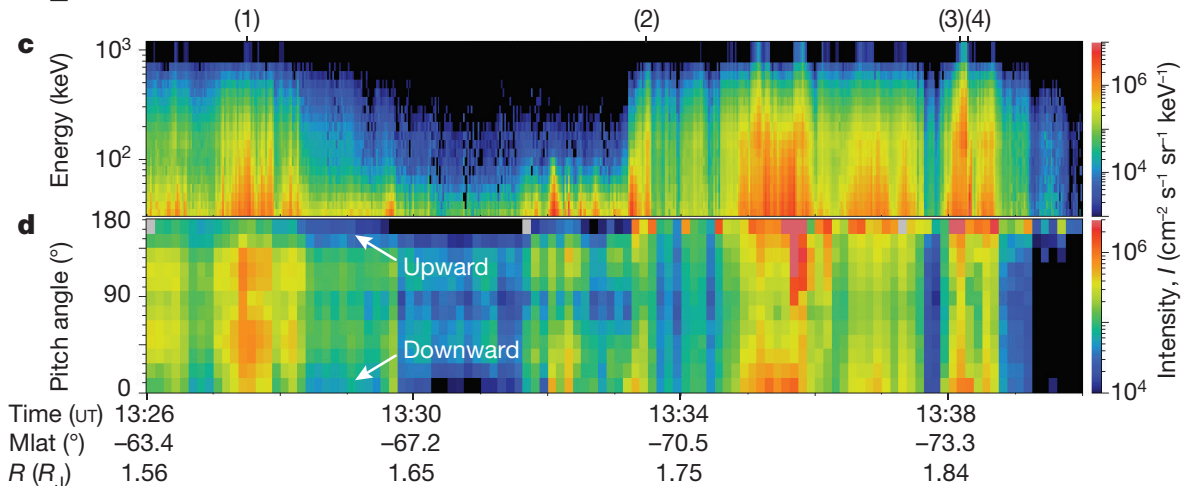

Figure 1 | Energetic electron data from JEDI during Juno's first perijove (PJ1) encounter with Jupiter (27 August 2016). a, Downward intensity $I$ versus electron energy $E$ spectra (see Methods) for the times identified in $\mathbf{c}$. b, Integrated downward electron energy flux, calculated as summarized elsewhere ${ }^{9}$ (see Methods). c, Intensity versus energy distributions for electrons within $20^{\circ}$ of the downward magnetic field direction. d, Pitch angle distributions of intensities averaged over electron energy (30-1,000 keV); the labels 'upward' and 'downward' indicate the portions of the plot (top and bottom) that represent electrons moving away from and towards Jupiter, respectively. The pitch angle is the angle between the particle velocity and the local magnetic field from the MAG instrument ${ }^{21}$. 'Mlat' is the dipole magnetic latitude using the VIP4 field model dipole $^{22} . R$ is the distance of Juno from the centre of Jupiter, in units of Jovian radius $\left(R_{\mathrm{J}}\right)$. The counting rates for these electron measurements are very high, so statistical error bars would not be visible on these plots.

${ }^{1}$ The Johns Hopkins University Applied Physics Laboratory, Laurel, Maryland, USA. ${ }^{2}$ Southwest Research Institute, San Antonio, Texas, USA. ${ }^{3}$ Jet Propulsion Laboratory, Pasadena, California, USA. ${ }^{4}$ Instituto Nazionale di Astrofisica-Instituo di Astofisica e Planetologia Spaziali, Roma, Italy. ${ }^{5}$ Physics and Astronomy Department, University of Texas at San Antonio, San Antonio, Texas, USA. ${ }^{6}$ University of Colorado, Boulder, Colorado, USA. ${ }^{7}$ Université de Liège, Technologies and Astrophysics Research Institute, Laboratoire de Physique Atmosphérique et Planétaire, Liège, Belgium. ${ }^{8}$ NASA Goddard Space Flight Center, Greenbelt, Maryland, USA. ${ }^{9}$ University of lowa, lowa City, lowa, USA. ${ }^{10}$ Princeton University, Princeton, New Jersey, USA. 


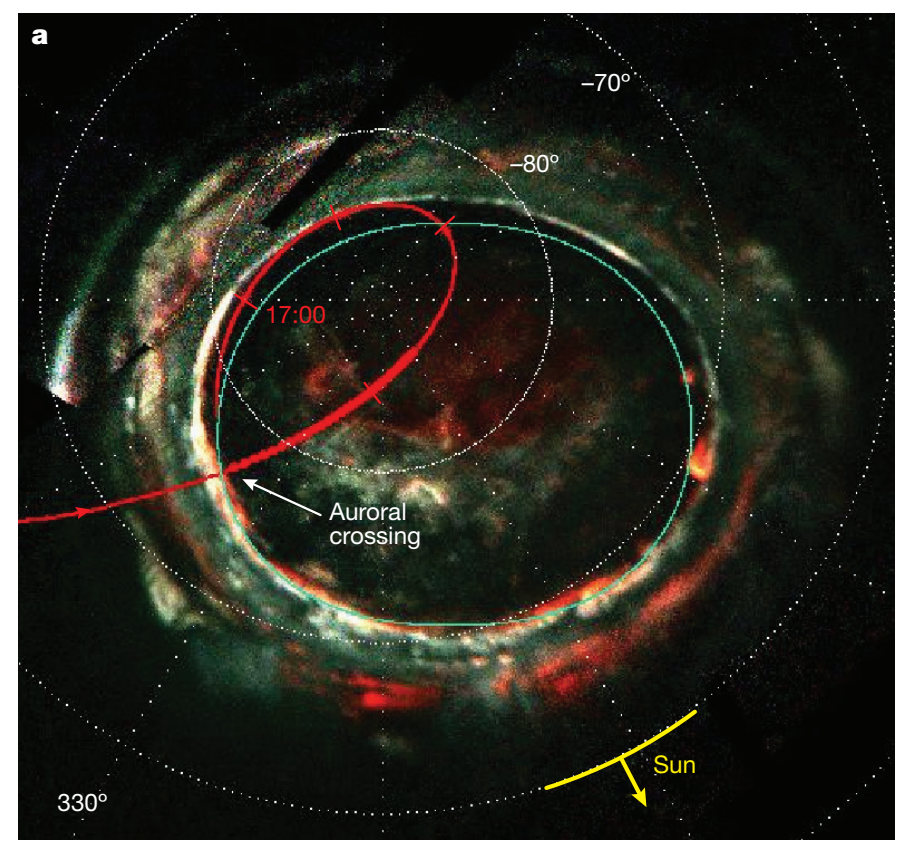

Figure 2 | Ultraviolet auroral images of Jupiter from the Juno Ultraviolet Spectrograph (UVS) instrument. The images contain intensities from three spectral ranges, false-coloured red, green and blue, providing qualitative information on precipitating electron energies (high, medium and low, respectively ${ }^{23}$. An estimate of the magnetic projections of the Juno trajectory is shown (red lines), determined using the VIPAL ${ }^{24}$ magnetic field model with large uncertainties, with tick marks in steps of $1 \mathrm{~h}$ (see Methods). The short yellow arcs with arrows indicate

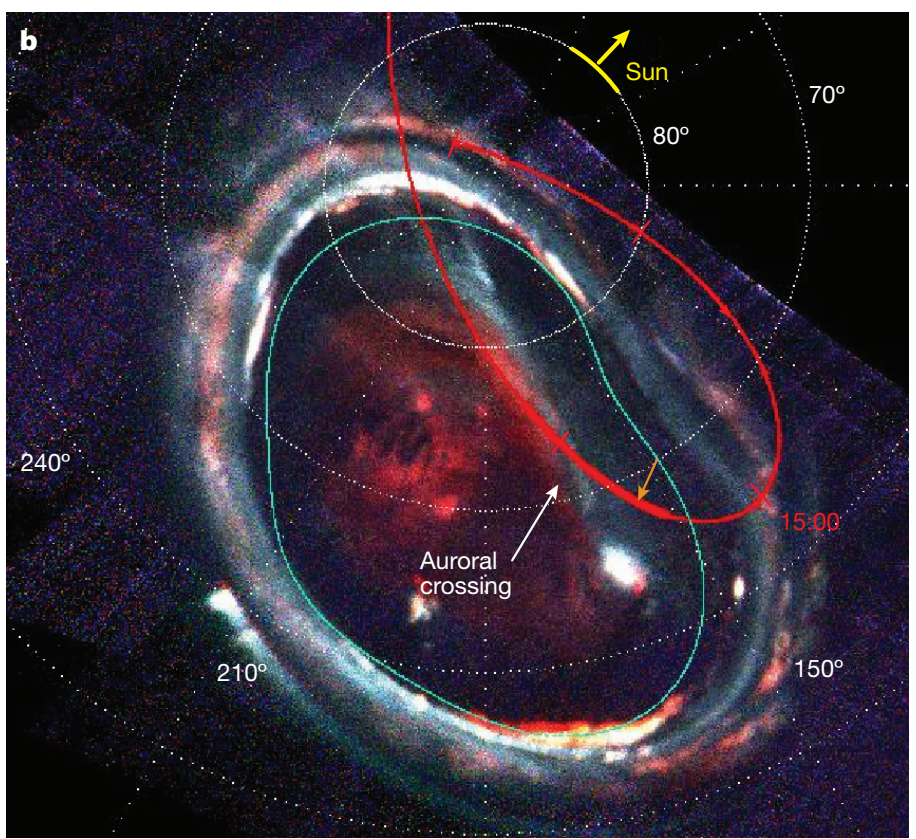

the direction to the Sun when the image was taken. The blue-green lines are the average positions of the main ultraviolet aurora for the south and north, respectively ${ }^{12,13}$. a, Jupiter's southern aurora taken during the fourth perijove (PJ4) encounter on 2 February 2017. b, Jupiter's northern aurora taken during the third perijove (PJ3) encounter on 11 December 2016. The single orange arrow indicates approximately when the particle data were taken (Fig. 3) and is discussed in Methods in the context of magnetic mapping errors.

a

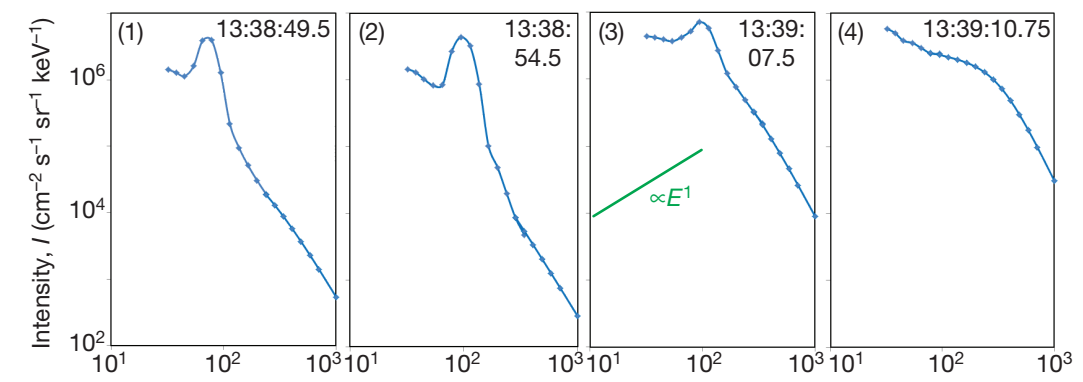

b
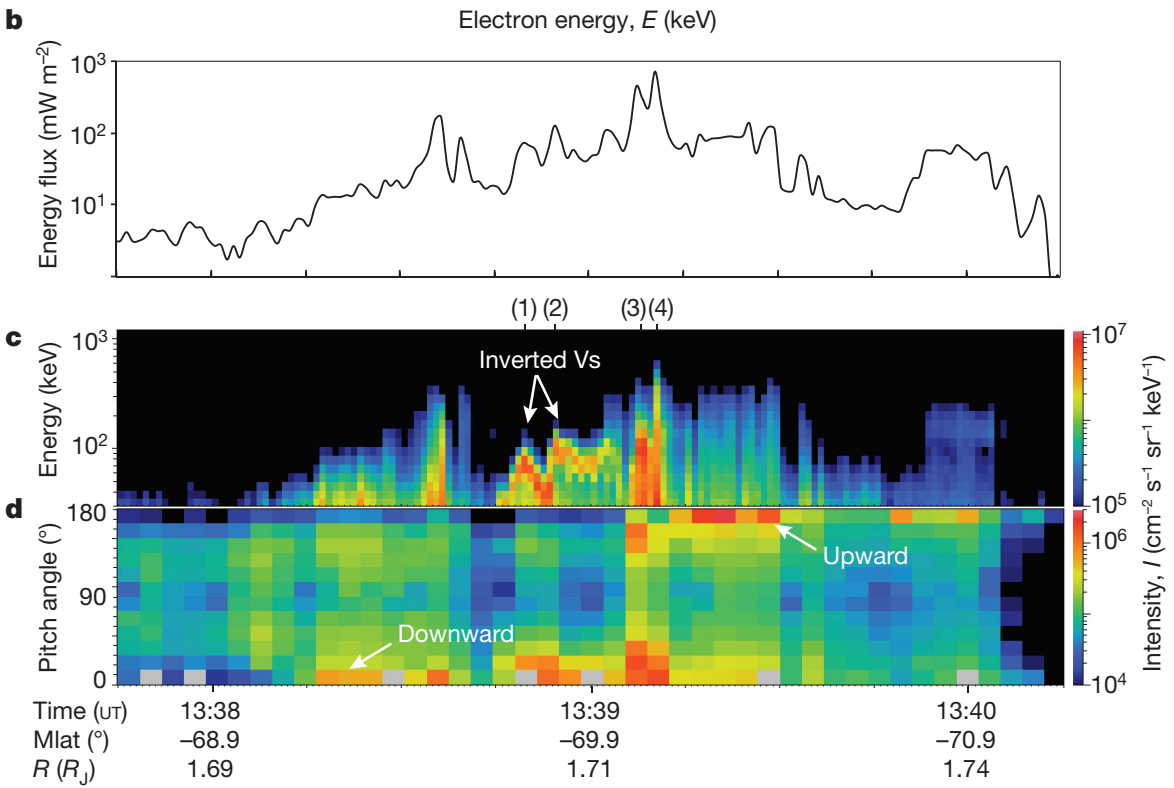

Figure 3 | Energetic electron data during Juno's fourth perijove (PJ4) encounter with Jupiter's low-altitude polar regions (2 February 2017).

As Fig. 1; the auroral context is shown in Fig. 2a. 



Figure 4 | Energetic electron data during Juno's third perijove (PJ3) encounter with Jupiter's low-altitude polar regions (11 December 2016). As Fig. 1; the auroral context is shown in Fig. 2 b.

perturbations were far weaker and more disordered than anticipated or modelled on the basis of the expected magnetic-field-aligned auroral electric currents ${ }^{8}$. Also, the downward electron energy spectra that Juno observed over the aurora did not have the anticipated strong energy peaks and, in particular, the expected peaks at energies of more than $50 \mathrm{keV}$ (refs 9, 10). Electron distributions with high-energy peaks were expected on the basis of the physics derived for Earth and the characteristic energies (50-500 keV) inferred from remote spectrographic imaging of Jupiter's aurora ${ }^{12-14}$.

First studies of Jupiter's high-energy ( $>30 \mathrm{keV}$ ) auroral electrons using the Jupiter Energetic Particle Detector Instrument (JEDI) ${ }^{15}$ used relatively low-resolution measurements $(30 s)^{9}$. However, the higher-time-resolution measurements $(0.5-1.5 \mathrm{~s})$ that we present here confirm the fundamental finding from the PJ1 encounter that no downward discrete auroral acceleration to energies of more than $30 \mathrm{keV}$ was observed (Fig. 1; the global auroral context is presented elswhere ${ }^{16}$, but is similar to Fig. 2a). In Fig. 1, downward energy fluxes are shown (peaking near about $700 \mathrm{~mW} \mathrm{~m}^{-2}$ ) that are sufficient to account for the nominal and intense auroral intensities that are observed remotely ${ }^{12,13,16}$. Surprisingly, even with the large magnitude of the downward energy fluxes, the upward energy fluxes are often even larger (these auroral distributions are often in the form of asymmetric, bi-directional angle beams; Fig. 1d). The downward electron intensity spectra shown in Fig. 1a reveal broad energy distributions rather than sharply peaked ones as anticipated from observations of Earth's aurora. It has been proposed that a broadband, stochastic acceleration is responsible ${ }^{9}$. The electrons measured at lower energies by the Jupiter Auroral Distribution Experiment (JADE) ${ }^{17}$ on Juno had similar features with no sharply peaked electron distributions ${ }^{10}$. Together JADE and JEDI measure the energies from $0.1 \mathrm{keV}$ to $1,000 \mathrm{keV}$ and thus show that no peaked electron distributions existed for the time period shown in Fig. 1 or for any time during the PJ1 encounter.

More recent perijove passes have revealed the presence of highenergy $(>50 \mathrm{keV})$, discrete, downward auroral acceleration in the form of 'inverted V' structures that are embedded in other ongoing processes, for about $50 \%$ of the main auroral crossings (Fig. 3; from the fourth perijove (PJ4) encounter; Fig. 2a shows the qualitative global auroral context). The intensity $(I)$ versus energy $(E)$ spectra show energy beams with positive slopes and that increase faster than $E^{+1}$ (a true energy beam has a positive slope in the phase-space density with a shape of $I / E$ in the non-relativistic regime ${ }^{18}$; see Methods). The peak in the intensity distributions is inferred to represent the electric potentials along magnetic field lines that have coherently energized the electrons ${ }^{2}$. For the clean inverted-V distributions, the intensities decrease rapidly above the energy peak. However, spectrum (3) in Fig. 3a might represent a transition from one process to another, or just the simultaneous occurrence of the two different processes. The peak in this intensity spectrum is marginally suggestive of a discrete acceleration process. However, a larger portion of downward energy flux comes from broadband additions, extending to energies both below and above the peaked structure. The highest downward energy fluxes come from spectrum (4) in Fig. 3a, which displays no evidence of discrete auroral acceleration.

One example of particularly energetic downward acceleration was observed during a northern polar pass within an arc-like structure located poleward of the nominal main auroras (Fig. 2b). Contrary to the case of the PJ4 encounter, such a location does not map to the middle magnetosphere but far into the outer magnetosphere ${ }^{19}$. Within this structure a downward electron beam was observed that peaked at about $400 \mathrm{keV}$, more than an order of magnitude larger than the largest 
values observed at Earth (Fig. 4) ${ }^{11}$. In this extreme case the downward energy fluxes (approximately $70 \mathrm{~mW} \mathrm{~m}^{-2}$ ) were more comparable in magnitude with the maximum downward energy fluxes that were observed elsewhere for this region $\left(150-300 \mathrm{~mW} \mathrm{~m}^{-2}\right.$; the entire event is not shown). However, even here the coherent discrete auroral acceleration was not the prime acceleration mechanism.

At Earth, the broadband or Alfvénic aurora represents stochastic acceleration that is caused by particle interactions with various downward-propagating Alfvén waves ${ }^{3,4}$ at radial positions of 4-5 Earth radii ${ }^{20}$, generating transitory aurora at the poleward boundary of the auroral oval. At low altitudes the downward fluxes will be stronger than the upward fluxes ${ }^{4}$, opposite to what is often, although not always, observed at Jupiter.

Another form of stochastic acceleration at Earth is the mostly upward acceleration that occurs in regions of downward electric currents $^{1,2}$. Much weaker downward components are revealed, cutting off at much lower energy than does the upward component. These downward fluxes are exceedingly weak, yielding 'black aurora'. These regions have qualitative similarities to those observed at Jupiter. But at Jupiter the downward intensities extend in energy to very high energies $(>1,000 \mathrm{keV})$, and have sufficient downward energy fluxes to power the most intense auroral emissions in the Solar System. We do not know whether the similarities are coincidental or result from similar physical processes. The magnetic perturbations are too weak and disordered to allow for decisions on the basis of electric current polarity ${ }^{8}$.

The transition in Fig. 3a from the coherent acceleration regions (spectra (1) and (2)), through spectrum (3), which combines stochastic and coherent acceleration, to the region dominated by stochastic acceleration (spectrum (4)) is intriguing. It is possible that auroral acceleration begins as a coherent discrete acceleration process like Earth's, but then instability is initiated as the drivers become stronger, resulting in the broadening of the distributions through stochastic processes. This and other possibilities will be pursued in future studies as additional data becomes available.

Online Content Methods, along with any additional Extended Data display items and Source Data, are available in the online version of the paper; references unique to these sections appear only in the online paper.

\section{Received 9 May; accepted 26 June 2017.}

1. Ergun, R. E. et al. FAST satellite observations of electric field structures in the auroral zone. Geophys. Res. Lett. 25, 2025-2028 (1998).

2. Carlson, C. W. Pfaff, R. F. \& Watzin, J. G. The Fast Auroral SnapshoT (FAST) mission. Geophys. Res. Lett. 25, 2013-2016 (1998).

3. Chaston, C. C. et al. The turbulent Alfvénic aurora. Phys. Rev. Lett. 100, 175003 (2008).

4. Amm, O. et al. Chapter 4: in situ measurements in the auroral plasma. Space Sci. Rev. 103, 93-208 (2002).

5. Cowley, S. W. H. \& Bunce, E. J. Origin of the main auroral oval in Jupiter's coupled magnetosphere-ionosphere system. Planet. Space Sci. 49, 1067-1088 (2001).

6. Hill, T. W. The Jovian auroral oval. J. Geophys. Res. 106, 8101-8107 (2001)

7. Ray, L. C., Ergun, R. E., Delamere, P. A. \& Bagenal, F. Magnetosphere-ionosphere coupling at Jupiter: effect of field-aligned potentials on angular momentum transport. J. Geophys. Res. 115, A09211 (2010).

8. Connerney, J. E. P. et al. Jupiter's magnetosphere and aurorae observed by the Juno spacecraft during its first polar orbits. Science 356, 826-832 (2017).

9. Mauk, B. H. et al. Juno observations of energetic charged particles over Jupiter's polar regions: analysis of monodirectional and bidirectional electron beams. Geophys. Res. Lett. 44, 4410-4418 (2017)

10. Allegrini, F. et al. Electron beams and loss cones in the auroral regions of Jupiter. Geophys. Res. Lett. 44, http://doi.org/10.1002/2017GL073180 (2017).
11. Arnoldy, R. L. in Physics of Auroral Arc Formation (eds Akasofu, S.-I. \& Kan, J. R.) 56-66 (AGU, 1981)

12. Gérard, J.-C. et al. Mapping the electron energy in Jupiter's aurora: Hubble spectral observations. J. Geophys. Res. 119, 9072-9088 (2014).

13. Gustin, J. et al. Characteristics of north Jovian aurora from STIS FUV spectral images. Icarus 268, 215-241 (2016).

14. Tao, C. et al. Variation of Jupiter's aurora observed by Hisaki/EXCEED: 2. Estimation of auroral parameters and magnetospheric dynamics. J. Geophys. Res. 121, 4055-4071 (2016)

15. Mauk, B. H. et al. The Jupiter Energetic Particle Detector Instrument (JEDI) investigation for the Juno mission. Space Sci. Rev. http://doi.org/10.1007/ s11214-013-0025-3 (2013)

16. Bonfond, B. et al. Morphology of the UV aurorae Jupiter during Juno's first perijove observations. Geophys. Res. Lett. 44, 4463-4471 (2017).

17. McComas, D. J. et al. The Jovian Auroral Distributions Experiment (JADE) on the Juno mission to Jupiter. Space Sci. Rev. http://doi.org/10.1007/ s11214-013-9990-9 (2013).

18. Krall, N. A. \& Trivelpiece, A. W. Principles of Plasma Physics Ch. 3 (McGraw-Hill, 1973).

19. Grodent, D. et al. Jupiter's polar auroral emissions. J. Geophys. Res. 108, 1366 (2003)

20. Janhunen, J., Olsson, A., Russell, C. T. \& Laakso, H. Alfvénic electron acceleration in aurora occurs in global Alfvén resonosphere region. Space Sci. Rev. 122, 89-95 (2006).

21. Connerney, J. E. P. et al. The Juno magnetic field investigation. Space Sci. Rev https://doi.org/10.1007/s11214-017-0334-z (2017).

22. Connerney, J. E. P., Acuña, M. H., Ness, N. F. \& Satoh, T. New models of Jupiter's magnetic field constrained by the lo flux tube footprint. J. Geophys. Res. 103, 11929-11939 (1998).

23. Gladstone, G. R. et al. The ultraviolet spectrograph on NASA's Juno mission. Space Sci. Rev. https://doi.org/10.1007/s11214-014-0040-z (2014).

24. Hess, S. L. G., Bonfond, B., Zarka, P. \& Grodent, D. Model of the Jovian magnetic field topology constrained by the lo auroral emissions. J. Geophys. Res. 116, A05217 (2011)

Acknowledgements We are grateful to NASA and contributing institutions that helped to make the Juno mission possible. This work was funded by NASA's New Frontiers Program for Juno via subcontract with the Southwest Research Institute.

Author Contributions B.H.M. is lead investigator for the Juno JEDI investigation, created Figs 1,3 and 4 and analysed the data. D.K.H. operated the JEDI instruments during the Juno polar passes. C.P. coordinated planning of the instruments for the Juno polar passes. G.C. determined the JEDI efficiencies for quantifying the intensities of the particle fluxes. P.K. determined the outof-band sensitivities of the JEDI instrument. A.M.R. developed the procedure for removing the minimum ionizing contamination of the electron spectra due to high-energy penetrators. S.J.B. is the principal investigator of the Juno mission. S.M.L. is a project scientist on the Juno mission. A.A. is lead investigator for the Juno Infra-Red Auroral Imager (JIRAM), helped to plan the Juno polar encounters and interpreted the auroral images. F.A. is a member of the Juno JADE plasmas sensor, helped to plan the Juno auroral encounters and interpreted the particle measurements. F.B. helped to plan the Juno polar encounters and performed the magnetic mapping of the Juno trajectory to the auroral atmosphere. B.B. is a member of the UVS imaging team and interpreted the Juno UVS auroral images. J.E.P.C. is lead investigator of the Juno magnetometer instrument and provided the magnetic field data necessary for ordering the particle data in Figs 1, 3 and 4. G.R.G. is lead investigator of the Juno UVS instrument and generated Fig. 2. W.S.K. is lead investigator of the Juno Waves investigation and helped to plan and interpret the observations of Jupiter's polar regions. D.J.M. is the lead developer of the Juno JADE plasma instrument and helped to interpret the electron measurements. P.V. is the lead investigator for the Juno JADE instrument, and helped to plan the Juno polar encounters and interpret the electron measurements.

Author Information Reprints and permissions information is available at www.nature.com/reprints. The authors declare no competing financial interests. Readers are welcome to comment on the online version of the paper. Publisher's note: Springer Nature remains neutral with regard to jurisdictional claims in published maps and institutional affiliations. Correspondence and requests for materials should be addressed to B.M. (Barry.Mauk@jhuapl.edu).

Reviewer Information Nature thanks J. Clarke, T. Cravens and the other anonymous reviewer(s) for their contribution to the peer review of this work. 


\section{METHODS}

Energy flux. Although summarized elsewhere ${ }^{9}$, here we provide additional information regarding the method of estimating the downward energy flux (in $\mathrm{mW} \mathrm{m}^{-2}$ ) of electrons that impinge onto Jupiter's atmosphere to generate aurora. The data are filtered for measurements that have pitch angles centred within $15^{\circ}$ of the downward magnetic field direction to estimate the average intensities in the geometric loss cone. The geometric loss cone is the cone that is centred on the magnetic field direction and contains the velocity vectors of those particles that will strike the atmosphere before the magnetic mirror force can reflect them back to the upward direction. We then perform the summation $\pi \sum_{n} I_{n} E_{n} \Delta E_{n}$ over all $n$, where $n$ represents the JEDI energy channels, and $I_{n}$ is the particle intensity from each channel, $E_{n}$ is the central energy of each channel and $\Delta E_{n}$ is the energy band pass of each channel. The coefficient $\pi$ is the area-projection-weighted size of the loss cone just above the atmosphere, used on the basis of the rough estimate from the observations that the downward geometric loss cones are fully populated. We assume that as the particles move along the magnetic field line from the spacecraft to the atmosphere there is no retarding electric field below the spacecraft that would decrease the energy of the electrons on their way to the atmosphere. Hence, the intensities in the loss cone just above the atmosphere are greater than or equal to the intensities at the spacecraft.

Energy spectra. The intensity versus energy spectra shown in Figs 1a, 3a and $4 \mathrm{a}$ were modestly corrected to take into account the fact that a portion of the higher-energy particles fully penetrate the detectors. A preliminary version of the correction procedure is summarized elsewhere ${ }^{9}$. These corrections do not change the fundamental character of the spectral shapes. The fractional penetration of the higher-energy particles yields an additional high-energy efficiency of the form

$$
\varepsilon=1-\exp \left[-2\left(\frac{480}{E_{\mathrm{keV}}}\right)^{3}\right]
$$

where $0<\varepsilon<1$ is the efficiency and $E_{\mathrm{keV}}$ is energy in kiloelectronvolts. Each electron that penetrates the detector leaves behind a fraction of its energy, called the 'minimum ionizing' energy. The distribution of minimum ionizing energy that is deposited is parameterized as

$$
\operatorname{MI}(E)=\frac{F_{1} F_{2}}{35.47}
$$

where

$$
\begin{aligned}
& F_{1}=\frac{1}{4}\left\{1+\tanh \left[0.1\left(E_{\mathrm{keV}}-112\right)\right]\right\}^{2} \\
& F_{2}=\exp \left(-\frac{E_{\mathrm{keV}}-85}{60}\right)
\end{aligned}
$$

and the factor of 35.47 factor normalizes the distribution to a unit area.

These equations are combined with a parameterized spectral function (equation (1) in ref. 25) to fit the higher energies and thereby reproduce the JEDI measured responses by means of a free-parameter optimization procedure. This process is visualized elsewhere ${ }^{9}$, but here the accuracy of the procedure (using the parameters of equations (1)-(3)) is substantially improved. The procedures described here are unnecessary for the spectra that have strongly peaked energy distributions, with the exception that the corrected efficiency factor (equation (1)) is used at the highest energies.

Magnetic mapping. Juno magnetic field measurements show that the configuration of the magnetic field close to the planet is substantially different from what prevailing magnetic field models can reproduce ${ }^{26}$. When an existing magnetic field model is used to map the position of the spacecraft onto the atmosphere, as was the case for Fig. 2, substantial uncertainties are expected. This point is illustrated here using Fig. $2 \mathrm{~b}$ and Extended Data Fig. 1. Extended Data Fig. 1 is identical to Fig. 4b-d except that the timescale is expanded to include the entire northern polar pass. The major bump that occurs in Extended Data Fig. 1a, centred at about 15:40 universal time (UT), occurs about half way (in terms of time) between the main auroral crossings near 15:00 UT and 16:20 UT. This feature clearly corresponds to the auroral arc structure in Fig. $2 \mathrm{~b}$ that appears in the central regions of the polar cap, poleward of the main auroral oval. Yet, the bump at 15:40 Ut occurs at a time along the projected trajectory in Fig. $2 \mathrm{~b}$ (indicated with a small orange arrow) that is well separated from the image of the arc. This problem is one of magnetic mapping; when an accurate model of the magnetic field close to the planet becomes available later in the Juno mission, we expect that the position of the spacecraft at 15:40 UT will map much more accurately to the position of the arc structure. This discussion is intended to illustrate why the auroral images at this point in time provide only qualitative global contexts to the in situ particle measurements. The images are also obtained at different times from those of mapped Juno crossings of the various features.

Phase space density. As detailed in standard text books ${ }^{18}$, the behaviour of statistical distributions of objects such as atoms or charged particles is governed by Liouville's theorem. When the interactions between the objects is weak (collisionless), Liouville's theorem simplies to the collisionless Boltzmann equation, known as the Vlasov equation when applied to electrified gases called plasmas. The Vlasov equation governs the behaviour of 'phase space density' (PSD) distributions, which are the densities of particles in the six-dimensional space comprising configuration space $(x, y, z)$ and momentum space $\left(p_{x}, p_{y}, p_{z}\right)$. $\operatorname{PSD}(p)$, where $p$ is the momentum vector, contains the signatures of acceleration processes on particle populations. The directional intensity of particle populations $(I(E))$ is generally used in observational studies because its shape better reflects the raw instrumental measurement parameters (compared to the PSD) and because it is useful in determining the important parameters of particle and energy flux onto a surface or atmosphere. But, the shape of directional intensity profiles can be misleading with regard to the processes that are acting on a population of particles. Such profiles can have peaked distributions even if there are no coherent acceleration processes acting on the populations (see, for example, Fig. 1a). There is a fairly easy conversion between the $I(E)$ and $\operatorname{PSD}(p): \operatorname{PSD}=I / p^{2}$, which in the non-relativistic regime is proportional to $I / E$. The clear identification of coherent acceleration of electrons by magnetic-field-aligned electric fields occurs only in the PSD, or equivalently in the $I(E)$ distributions with positive slopes that are greater in magnitude than the slope of $E^{m}$ with $m>1$.

Data availability. The data presented here are available from the Planetary Plasma Interactions Node of NASA's Planetary Data System (https://pds-ppi.igpp.ucla. edu/). All data are in the form of column-labelled, .csv, ASCII flat files.

25. Mauk, B. H. et al. Energetic ion characteristics and neutral gas interactions in Jupiter's magnetosphere. J. Geophys. Res. 109, A09S12 (2004).

26. Bolton, J. S. et al. Jupiter's interior and deep atmosphere: the initial pole-topole passes with the Juno spacecraft. Science 356, 821-825 (2017). 


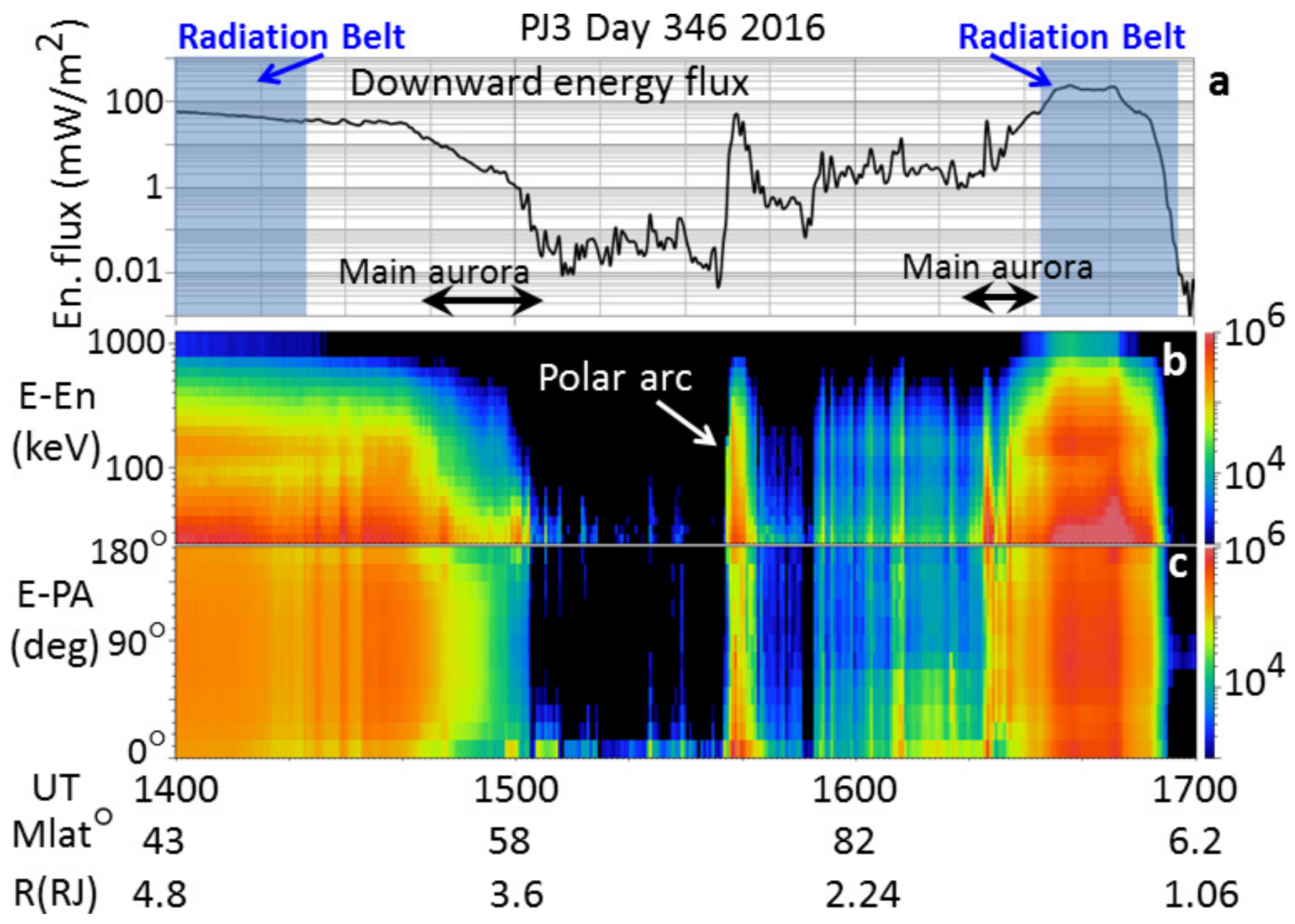

Extended Data Figure 1 | Energetic electron data during the PJ3 encounter with Jupiter's polar regions (11 December 2016). Same as Fig. $4 \mathrm{~b}-\mathrm{d}$, but showing a greatly extended period of time that encompasses

the entire northern polar pass. The horizontal brightening centred near $150 \mathrm{keV}$ in $\mathbf{b}$, particularly in the radiation belts, is the minimum ionizing feature discussed in Methods. 